Bundesgesundheitsbl 2015 · 58:1-3

DOI 10.1007/s00103-014-2102-5

Online publiziert: 13. Dezember 2014

(c) Springer-Verlag Berlin Heidelberg 2014

\author{
Uwe Koch-Gromus ${ }^{1}$. Hans-Jürgen Bartz ${ }^{2}$ \\ ${ }^{1}$ Dekan der Medizinischen Fakultät, Universitätsklinikum Hamburg-Eppendorf, Hamburg, Deutschland \\ ${ }^{2}$ Leitung GB QM und klinisches Prozessmanagement, Universitätsklinikum Hamburg-Eppendorf, \\ Hamburg, Deutschland
}

\title{
Patientensicherheit durch Risikomanagement
}

anderes als eine Formalisierung eines kollegialen Gesprächs. Hierbei steht die Reevaluation der medizinischen Information durch die Peer Reviewer im Vordergrund. Diese liefern gleichzeitig den Blick von außen im Sinne einer Zweitmeinung. Das Risiko für einen „Tunnelblick“ oder für „Fixierungsfehler" kann so minimiert werden. Ein wirksames klinisches Risikomanagement muss in den kontinuierlichen Verbesserungsprozess der „lernenden Organisation" integriert werden. Krankenhäuser sind nach der im April diesen Jahres veröffentlichten Richtlinie des Gemeinsamen Bundesausschusses über die grundsätzlichen Anforderungen an ein einrichtungsinternes Qualitätsmanagement verpflichtet, ein klinisches Risikomanagement einzurichten. Es kommt also in Zukunft noch stärker darauf an, das klinische Risikomanagement, wie es aus der täglichen klinischen Arbeit heraus bereits seit langem praktiziert wird, mit Hilfe moderner Methoden weiter zu entwickeln.

Das vorliegende Heft gibt in 14 Beiträgen einen aktuellen Überblick über Grundlagen und spezifische Instrumente zum Thema „Patientensicherheit durch Risikomanagement" in Deutschland. Im Eingangsbeitrag bilanziert M. Schrappe die insgesamt positive Entwicklung im Bereich Patientensicherheit 10 Jahre nach Gründung des „Aktionsbündnis Patientensicherheit". Vor allem die Einführung von Methoden zur Behebung „blinder Flecken“ (z. B. Critical Incident Reporting System, CIRS) und die Veränderung des Fokus von der Frage der individuellen Schuld auf Analysen von Organisationen, Teamfaktoren und Management-Strukturen werden von M. Schrappe positiv hervorgehoben. Des Weiteren verweist der Autor auf die Notwendigkeit, die Patienten- und Nutzenperspektive bei der Weiterentwicklung von Konzepten der Patientensicherheit stärker mit einzubeziehen.

Die beiden nachfolgenden Beiträge berichten über die Aktivitäten zum Thema Patientensicherheit jenseits der Landesgrenzen Deutschlands. S. Barth, B. Godschalk und G. Jonitz analysieren die Handlungsmöglichkeiten der Europäischen Kommission zur Förderung von Patientensicherheit und diskutieren den potentiellen Mehrwert, der auf EU-Ebene entstehen kann. Der Beitrag von $\mathbf{H}$. Rutberg, M. Eckhardt, O. Biermann beschreibt Infrastruktur, Methoden und einbezogene Partner in der Patientensicherheitsarbeit in Schweden sowie die Zusammenarbeit der nordischen Länder in diesem Themenfeld.

F. Wenzel und M. Steinmeister geben im nächsten Beitrag einen Überblick über das neue Patientenrechtegesetz (PatRG). Sie prüfen insbesondere die Frage, welche unmittelbaren Wirkungen die veränderten Regelungen zum Behandlungsvertrag auf die alltägliche Berufsausübung der Ärzte haben. Die Autoren diskutieren auch mögliche Risiken des PatRG für die Vertrauensbeziehung zwischen Arzt und Patient.

Die nächsten beiden Beiträge analysieren den potentiellen Nutzen von Leitlinien und Zertifizierungen für die Entwicklung der Patientensicherheit. C. Muche-Borowski, M. Nothacker und I. Kopp befassen sich mit der Frage, ob Leitlinien die Lücke zwischen Evidenz und Anwendung im alltäglichen Handeln in der medizinischen Versorgung schließen können. Sie bejahen diese Frage eindeutig, verweisen aber darauf, dass 
der potentielle Nutzen in starkem Maße von der Implementierbarkeit und Praktikabilität der Leitlinien abhängt. Insofern sei die Entwicklung einer stringenten Implementierungsstrategie und die Auswahl geeigneter, spezifischer Implementierungsmaßnahmen erforderlich. Diese haben das Ziel, die Versorgungsqualität durch dauerhaft leitliniengerechtes Verhalten zu verbessern.

R. Waßmuth diskutiert in seinem Beitrag den potentiellen Nutzen von Zertifizierungen auf das Risikomanagement und die Patientensicherheit. Dies geschieht dadurch, dass zentrale Anforderungen an die Behandlungsqualität erfüllt sind und Standards eingehalten werden. Relevante Determinanten einer Sicherheitskultur und das Vorgehen zur Korrektur und Vorbeugung von unerwünschten Ereignissen werden in Zertifizierungen abgebildet.

H.-J. Bartz versteht die systemische Fehleranalyse als zentralen Bestandteil des klinischen Risikomanagements. Dieses umfasst alle klinischen und administrativen Aktivitäten, die Schadensrisiken für die Organisation identifizieren, bewerten und reduzieren. Dies geschieht auf der Basis einer systemischen Analyse von Zwischenfällen und Fehlern. Die Bedingung für eine ergebnisorientierte systemische Fehleranalyse (z. B. Error \& RiskAnalysis) ist eine offene Unternehmenskultur, in der Fehler in einem sanktionsfreien Umfeld transparent gemacht werden. Der Fehleranalyse folgen dann wirksame Verbesserungsmaßnahmen.

S. Kluge und H. Bause gehen davon aus, dass ein freiwilliges „Peer-ReviewVerfahren" mehr Patientensicherheit in der Intensivmedizin schaffen kann. Weil im Hochrisikobereich der Intensivstation Fehler von besonderer Tragweite sein können, ist ein funktionierendes Qualitäts- und Risikomanagement ein besonders wichtiger Bestandteil der Behandlung von schwerkranken Patienten. Die Autoren schildern die Erfahrungen mit dem Instrument des freiwilligen „Peer Review“. Hierbei werden im kollegialen Umfeld auf der Intensivstation strukturiert zentrale Fragen zur Struktur-, Prozess- und Ergebnisqualität erörtert.

H. Schneider diskutiert die erheblichen Vorbehalte gegenüber einer flächen- deckenden Implementierung der elektronische Patientenakte (EPA) in Deutschland. Er sieht in ihr neben den Vorteilen, die sich aus einer schnellen Verfügbarkeit von Daten ergeben, auch ein besonders wirksames Instrument zur Steigerung der Patientensicherheit. Insbesondere elektronische Medikationssysteme können dies belegen. Auf der Basis von den seit 2009 gesammelten Erfahrungen mit der elektronischen Patientenakte am Universitätsklinikum Hamburg-Eppendorf demonstriert der Autor den Nutzen der EPA mit Fokus auf die Patientensicherheit.

M. Issleib und C. Zöllner beschreiben das simulationsbasierte Training von Ärzten und Pflegepersonal als ein sehr wirksames didaktisches Instrument zur Vorbereitung auf Notfälle in der klinischen Arbeit. Ein modernes Simulationstraining erlaubt es, seltene Komplikationen oder Notfallmaßnahmen außerhalb der unmittelbaren Patientenversorgung zu trainieren. Fehler können im simulationsbasierten Training erkannt und systematisch korrigiert werden. Auch die für die Zusammenarbeit besonders wichtigen Interaktionen im Team lassen sich hier einüben. Die Autoren beschreiben vor dem Hintergrund langjähriger eigener klinischer Erfahrungen belegbare Verbesserungen von Sicherheitskultur und Mitarbeiterzufriedenheit.

S. Neumann stellt die Bedeutung eines strukturierten Konfliktmanagements als Element des klinischen Risikomanagements heraus. Er sieht hierfür dann einen besonderen Bedarf, wenn die Analysen von Fehlern darauf hinweisen, dass ein interpersonaler Konflikt als Ursache oder beitragender Faktor in Betracht zu ziehen ist. Der Autor beschreibt das an der Europa-Universität Viadrina in Frankfurt an der Oder entwickelte Modell des strukturierten Konfliktmanagementsystems. Er hält es für einen sinnvollen und umsetzbaren Ansatz, eine bestehende Lücke im Methodenspektrum des klinischen Risikomanagements zu füllen.

Ebenfalls ein psychologisches Thema, wenn auch auf eine ganz andere Zielgruppe ausgerichtet, steht im Zentrum des Beitrages von D. L. B. Schwappach. Hier geht es um den Umgang mit Patienten, Ange- hörigen und dem involvierten Personal nach Behandlungsfehlern. Diese Behandlungsfehler, insbesondere solche mit Patientenschädigung, können für alle genannten Gruppen langanhaltende und belastende Konsequenzen haben. Insofern hat die angemessene Aufarbeitung der entstandenen Situation große Relevanz für die Betroffenen. Der Autor beschreibt einerseits die unterschiedlichen Reaktionen bei den Betroffenen, andererseits die Zielsetzungen und Arbeitsstrategien unterstützender Interventionen. Darüber hinaus behandelt er die in diesem Arbeitsprozess auftretenden Barrieren und Schwierigkeiten.

B. Hoffmann, H. Siebert und A. Euteneier fokussieren das Thema Patientensicherheit in der Ausbildung der Gesundheitsberufe. Die Autoren verweisen zunächst darauf, dass diese Aufgabe gesetzlich bisher unzureichend geregelt ist. Inzwischen liegen aber auch in Deutschland eine Reihe diesbezüglicher Curricula und Trainingskonzepten vor. Besondere Bedeutung komme dem Lernzielkatalog für Kompetenzen in der Patientensicherheit zu. Dieser geht auf eine Initiative des „Aktionsbündnis Patientensicherheit“ zurück. Er beschreibt für alle Gesundheitsberufe die erforderlichen Kenntnisse und Fertigkeiten für eine sichere Patientenversorgung. Der Lernzielkatalog kann als wichtige Orientierung dienen, wenn die Patientensicherheit als Ausbildungs- und vor allem auch Prüfungsgegenstand in den Berufsgesetzen für die Gesundheitsberufe verankert werden soll.

Der Beitrag von M. Müller schließt das Themenheft ab. Er beschreibt und analysiert die Rahmenbedingungen und Strategien des Risiko- und Fehlermanagement in der Luftfahrt. Dabei diskutiert er die Frage, ob die Medizin aus den hier geschaffenen Voraussetzungen und Ansätzen Nutzen ziehen kann. Der Autor kommt zu der klaren Einschätzung, dass sich die Sicherheitsstrategien der Luftfahrt in wesentlichen Komponenten auch auf die Medizin übertragen lassen und macht dies an Beispielen deutlich.

Wir wünschen den Leserinnen und Lesern eine spannende Auseinandersetzung mit den Beiträgen dieses Themenhefts. 


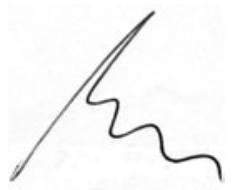

Prof. Dr. Dr. Uwe Koch-Gromus

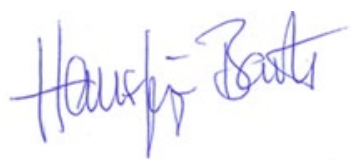

Dr. H.-J. Bartz

\section{Korrespondenzadressen}

\begin{tabular}{|c|c|}
\hline & $\begin{array}{l}\text { Prof. Dr. Dr. U. Koch-Gromus } \\
\text { Dekan der Medizinischen } \\
\text { Fakultät } \\
\text { Universitätsklinikum } \\
\text { Hamburg-Eppendorf } \\
\text { Martinistraße } 52 \\
20246 \text { Hamburg } \\
\text { koch@uke.uni-hamburg.de }\end{array}$ \\
\hline & $\begin{array}{l}\text { Dr. med. H.-J. Bartz, MBA } \\
\text { Leitung GB QM und klinisches } \\
\text { Prozessmanagement } \\
\text { Universitätsklinikum } \\
\text { Hamburg-Eppendorf } \\
\text { Martinistr 52, Gebäude } 013 \\
\text { 20246 Hamburg } \\
\text { h.bartz@uke.de }\end{array}$ \\
\hline
\end{tabular}

